\title{
Extensive Resection for Treatment of Locally Advanced Primary Mucinous Adenocarcinoma Arising From Fistula-in-Ano
}

\author{
Jordan Au, Francis M. Hulme-Moir, Andrew Herd, Mathew A. Kozman \\ Department of General Surgery, North Shore Hospital, Auckland, New Zealand
}

We report a case of a 66-year-old male who presented with a locally advanced primary mucinous adenocarcinoma arising from a fistula-in-ano. The presentation was typical for perianal sepsis and fistula-in-ano with anal pain and chronic discharge. Initial treatments with fistula debridement and seton were performed. Subsequent review of histology revealed underlying adenocarcinoma, while magnetic resonance imaging (MRI) showed local invasion into the prostate. The patient received neoadjuvant chemoradiotherapy followed by pelvic exenteration to maximize the chance of achieving cure. Features of this case are discussed together with its implications, including treatment guidelines and typical MRI findings.

Keywords: General surgery; Colorectal surgery; Rectal fistula; Mucinous adenocarcinoma

\section{INTRODUCTION}

Perianal sepsis and development of fistula-in-ano are common surgical problems, often having a significant impact on patients' quality of life and proving challenging to manage. Rare cases may be associated with malignancy, with the fistula-in-ano either arising as a result of a primary anorectal malignancy, or primary fistula tract malignancy occurring as a consequence of the prolonged inflammatory nature of the fistula. Thus, we report a rare and interesting case of locally advanced mucinous adenocarcinoma arising from fistula-in-ano. In addition, we discuss the decision-making process and management pathway adopted.

\section{CASE REPORT}

This study was approved by the Institutional Review Board of

Received: Sep 15, 2019 - Revised: Nov 17, 2019 - Accepted: Nov 19, 2019 Correspondence to: Jordan Au, MB.ChB.

Department of General Surgery, North Shore Hospital, 15 Shea Terrace, Takapuna, Auckland 0622, New Zealand

Tel: +64-2102245097

E-mail: jordanauj@gmail.com

ORCID: https://orcid.org/0000-0002-9455-069X

(C) 2021 The Korean Society of Coloproctology

This is an open-access article distributed under the terms of the Creative Commons Attribution NonCommercial License (https://creativecommons.org/licenses/by-nc/4.0) which permits unrestricted noncommercial use, distribution, and reproduction in any medium, provided the original work is properly cited.
North Shore Hospital (Auckland, New Zealand). Informed consent was gained from the patient prior to writing.

A 66-year-old male presented for treatment of fistula-in-ano. His medical history was significant for type II diabetes mellitus and previous ankle fracture. He had a 25 pack-year smoking history and his only medication is metformin. Over the 4 months preceding this presentation, he suffered recurrent perianal sepsis requiring multiple incision and drainage procedures. Examination of the perineum showed extensive scarring and multiple external openings on the left side, extending toward the left buttock. Ongoing purulent discharge was noted.

Pelvic magnetic resonance imaging (MRI) showed a complex tract in the left ischioanal fossa with an extension superiorly toward the left-lateral margin of the prostate. T2 high signal intensity was seen within the tracts (Fig. 1).

An examination under anesthetic was performed, the fistula was debrided and a seton was placed. Histopathology of the tissue debrided revealed anal mucosa with underlying infiltrating mucinous adenocarcinoma extending into the ischiorectal adipose tissue. The tumor was DNA mismatch repair proficient and BRAF wild-type on immunohistochemistry. It was cytokeratin (CK) 20, caudal type homeobox 2, and CK19 positive, but negative for CK7, GATA 3, estrogen receptor, and progesterone receptor. Computed tomography staging showed no distant metastasis to chest or abdomen. Subsequent colonoscopy showed no visible mucosal primary lesion (Fig. 2). 


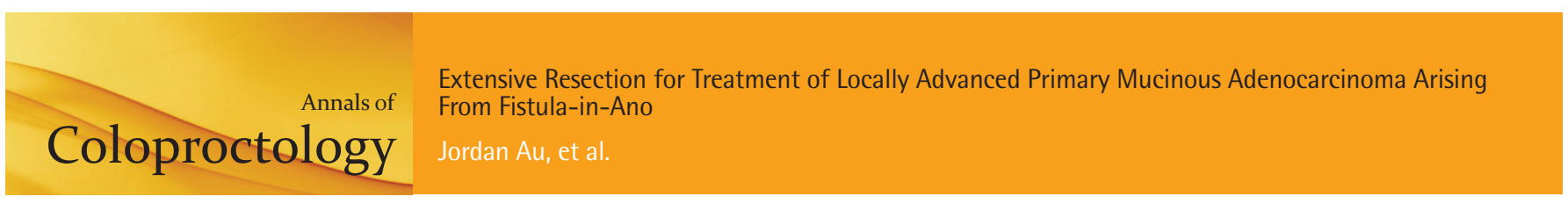

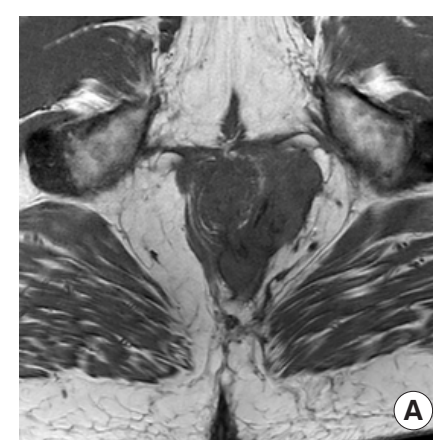
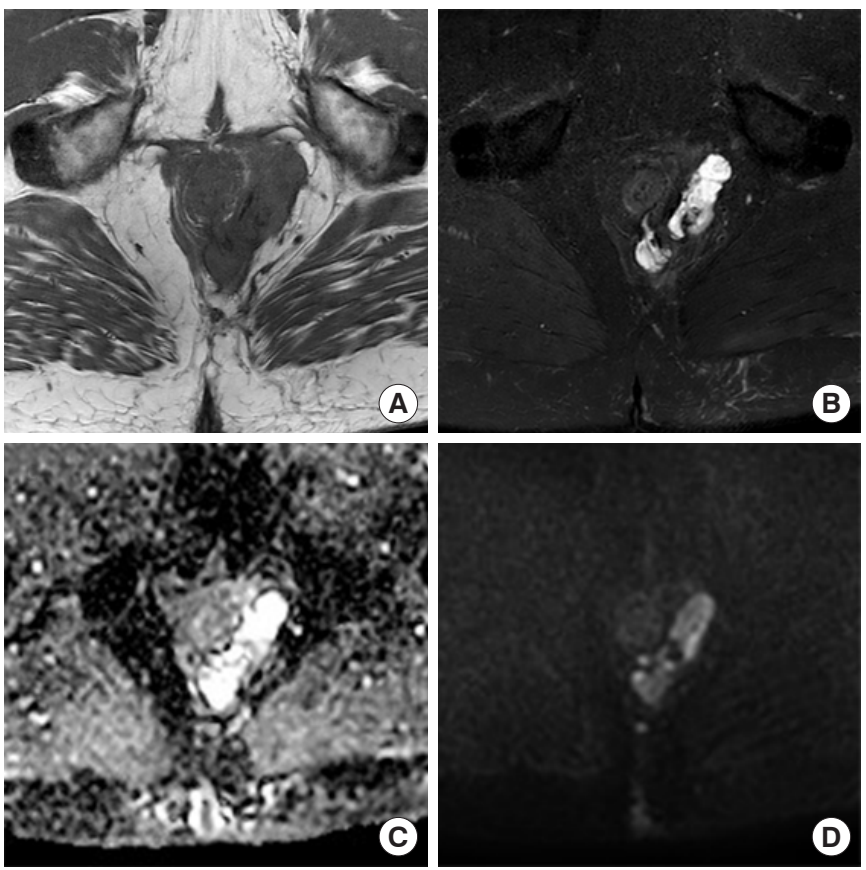

Fig. 1. Magnetic resonance imaging pelvis showing axial T1 and T2 fat-suppressed images (A, B), with granular appearance and lack of thick fibrous capsule around mucin pools. (C, D) Diffusion-weighted imaging showing restricted diffusion, but apparent diffusion coefficient reveals a relatively nonrestricted pattern.
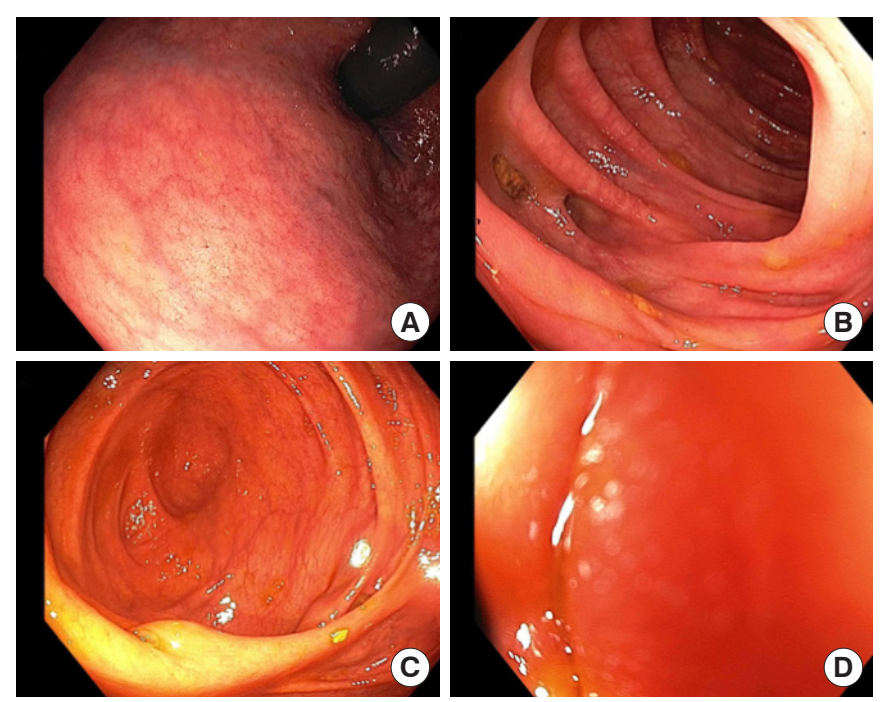

Fig. 2. Colonoscopic images showing rectum (A), sigmoid colon with diverticulosis (B), caecum and ileocaecal valve (C), and terminal ileum (D).

Decision regarding progression of management was based on a number of factors discussed in the context of a colorectal cancer multidisciplinary team meeting. Namely, confirmation of adeno-
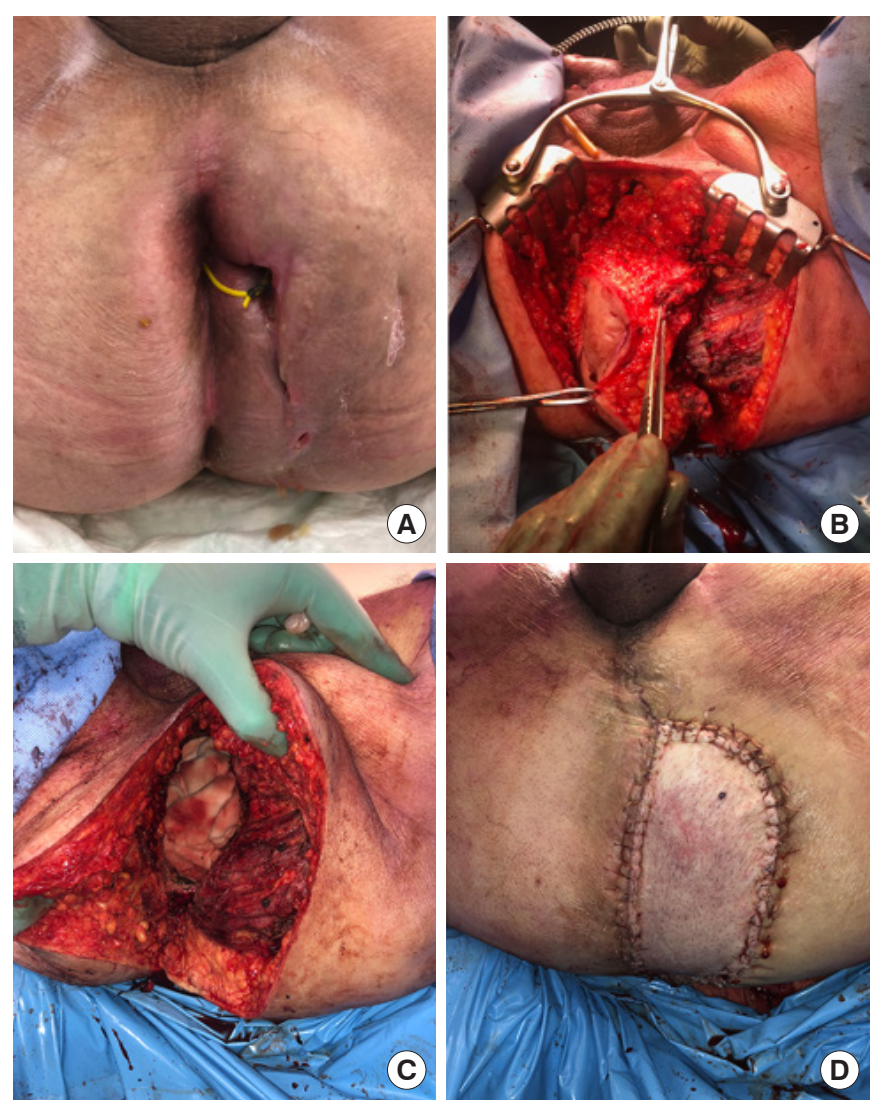

Fig. 3. Intraoperative images showing perineum with seton in place (A), resection of the tumor and surrounding structures $(B, C)$, and flap reconstruction (D).

carcinoma upon biopsy, the absence of distant metastasis on staging, and the presence of prostatic invasion on MRI imaging. As such, it was felt that administration of neoadjuvant chemoradiotherapy followed by pelvic exenteration was the only viable management option to achieve potential cure.

He commenced neoadjuvant chemoradiotherapy with capecitabine, completing 50 Gy over 25 fractions. Repeat MRI showed no significant response. He proceeded to pelvic exenteration with abdominoperineal resection (APR), uretherectomy, cystoprostatectomy with ileal conduit formation, partial excision of left obturator internus, S5 sacrectomy, and excision of medial head of gluteus maximus. The perineum was reconstructed using a transverse rectus abdominus musculocutaneous flap (Fig. 3).

Histology showed a well-differentiated mucinous adenocarcinoma arising from a fistula-in-ano, confined to the surrounding tissues (Fig. 4). No anorectal mucosal involvement was evident. The component of the fistula-in-ano adjacent to the prostate was free of malignancy. No regional lymph node metastases were identified.

Postoperatively he developed a pelvic collection which was treated with radiologically-guided drainage and intravenous anti- 

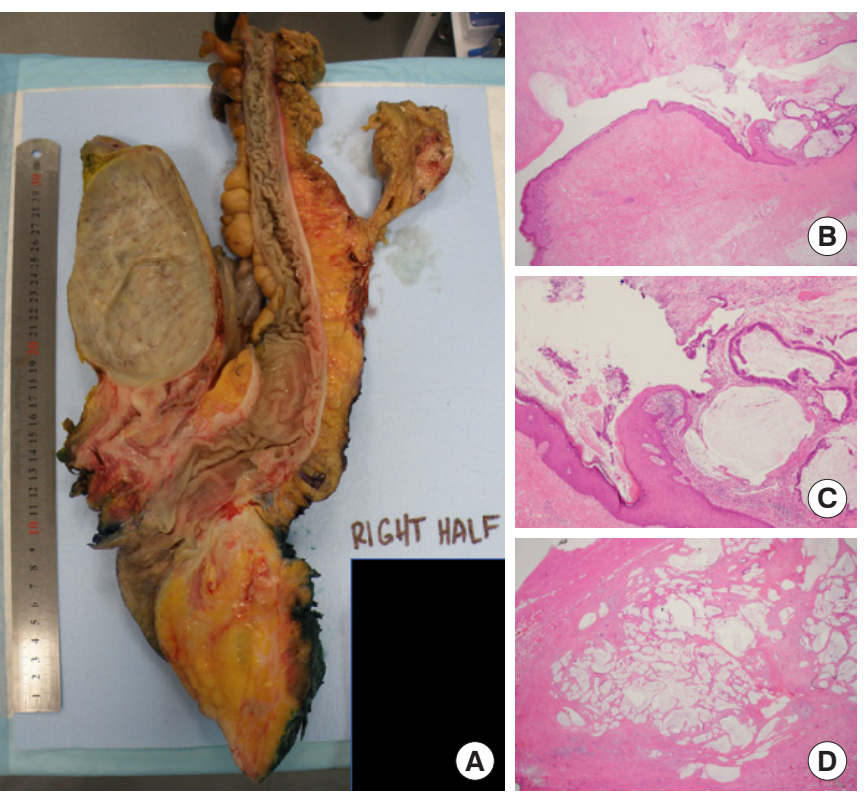

(B)

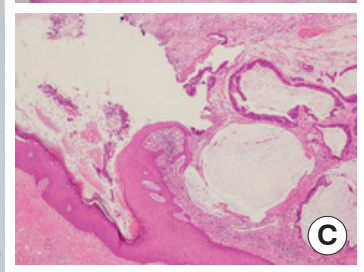

A

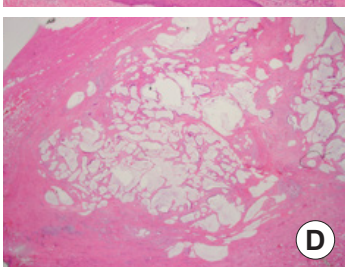

Fig. 4. Macroscopic image of pelvic exenteration specimen (A) and histopathology slides (B-D, H\&E). (B) Low-power image of the fistula-in-ano and tumor. (C) High-power image at the junction of the skin and the tumor. (D) High-power image of the tumor within the skeletal muscle.

biotics. Otherwise, his recovery was uneventful. Multidisciplinary team decision was for adjuvant chemotherapy.

\section{DISCUSSION}

Primary mucinous adenocarcinoma of the perianal region is an exceedingly rare condition [1]. A subset of these are found to be associated with chronic fistula-in-ano making early diagnosis difficult. As Rosser first established in 1934, these cancers are often associated with a prolonged history of chronic fistula disease greater than 10 years [2]. Recently, however, cases where the fistula has been present for less time are being reported, in some cases being diagnosed after 2 months [3]. In this particular case, mucinous adenocarcinoma was diagnosed only 4 months after the fistula. The histopathology findings of the exenteration specimen suggest that this was a primary adenocarcinoma originating within the fistula tract, as no anorectal mucosal involvement was evident. However, it is peculiar that this occurred in the context of a relatively short history of fistula-in-ano (4 months), as primary fistula tract tumors would typically require a prolonged period of inflammation to prompt metaplastic change [4].

The MRI findings are nonspecific. The most common finding is hyperintense content on T2 imaging, representing mucin pooling [5]. This can be difficult to distinguish from an abscess; however, this malignancy tends to show heterogeneity with a grape-like appearance [5]. No thick fibrous capsule is seen [5]. Diffusionweighted imaging (DWI) showed increased signal intensity in keeping with pus; however, the apparent diffusion coefficient showed increased signal intensity, likely representing less restricted diffusion of mucin vs. pus with T2 shine-through confusing the DWI image [6] (Fig. 1).

Literature reviewed revealed similar cases of adenocarcinoma arising from fistula-in-ano being treated with APR. These suggest that most cases are locally aggressive, with a low risk of lymph node involvement or distant metastasis [1]. However, evidencebased treatment guidelines are limited. Anecdotally, APR may be effective for nonmalignant localized disease. Chemoradiotherapy may be beneficial but there is a lack of data to support or deny its use. Some have reported successful treatment of local disease with surgery only [7]. In our case, there was no significant response to chemoradiotherapy, while others report significant reduction in tumor size [5]. Given the current uncertainty, our multidisciplinary team opted for neoadjuvant therapy to maximize likelihood of cure.

This case was unique for several reasons. First, mucinous adenocarcinoma arising within a fistula-in-ano is a rare occurrence. Second, the fistula-in-ano associated with this primary malignancy tracked via a complex course toward and abutting the prostate, raising concern of local invasion into the prostate. As such, multidisciplinary decision was for more radical resection to acquire clear margins and maximize the likelihood of cure. To our knowledge, this is the first case where tracking of the fistula-inano associated with malignancy was so extensive, thus warranting a more radical resection to ensure complete resection. Third, this case emphasizes the limitations of preoperative imaging and examination under anesthetic in ascertaining the extent of malignant involvement in the presence of a fistula-in-ano.

This case presentation highlighted the difficulties associated with diagnosis, staging, and treatment of primary malignancies arising from within a fistula-in-ano. Guidance of a multidisciplinary team is imperative in this condition, due to challenging interpretation of preoperative imaging to assess extent of tumor involvement and sparsity of evidence to guide treatment decisions.

\section{CONFLICT OF INTEREST}

No potential conflict of interest relevant to this article was reported.

\section{REFERENCES}

1. Purkayastha A, Sharma N, Dutta V, Bisht N, Pandya T. Mucinous adenocarcinoma of perianal region: an uncommon disease treated with neo-adjuvant chemo-radiation. Transl Gastroenterol Hepatol 2016;1:52.

2. Alvarez-Laso CJ, Moral S, Rodríguez D, Carrocera A, Azcano E, Cabrera A, et al. Mucinous adenocarcinoma on perianal fistula. A rising entity? Clin Transl Oncol 2018;20:666-9. 


\section{Coloproctology Jordan Au, et al.}

3. Yamada K, Miyakura Y, Koinuma K, Horie H, Lefor AT, Yasuda Y, et al. Primary and secondary adenocarcinomas associated with anal fistulae. Surg Today 2014;44:888-96.

4. Chowdri AN, Fazl NS, Parray Q, Wani AR, Thakur N. Fistula in ano and carcinoma: primary or secondary? Clin Surg 2016;1:1062.

5. Hama Y, Makita K, Yamana T, Dodanuki K. Mucinous adenocarcinoma arising from fistula in ano: MRI findings. AJR Am J Roentgenol 2006;187:517-21.
6. Ho CM, Tan CH, Ho BC. Clinics in diagnostic imaging (143). Perianal mucinous adenocarcinoma arising from chronic fistulain-ano. Singapore Med J 2012;53:843-9.

7. Chrysikos D, Mariolis-Sapsakos T, Triantafyllou T, Karampelias V, Mitrousias A, Theodoropoulos G. Laparoscopic abdominoperineal resection for the treatment of a mucinous adenocarcinoma associated with an anal fistula. J Surg Case Rep 2018;2018:rjy036. 